\title{
Electrical conductivity of viscous liquid foods
}

\author{
Balaji Subbiah, Ken R. Morison
}

Department of Chemical and Process Engineering, University of Canterbury, Christchurch, New Zealand

\begin{abstract}
The electrical conductivities of foods are used for quality assurance, electrical tomography studies and are required for effective simulation of electrical heating processes such as ohmic and microwave heating. Solutions containing milk solids, whey proteins, sugars and sodium carboxymethyl cellulose (NaCMC), with and without electrolytes were prepared and tested. The electrical conductivity was measured using an RCL meter connected to a parallel plate probe. At low concentrations the conductivity increased with concentration, but in some viscous solutions the reduced ion mobility caused a drop in conductivity. The conductivity of sugar solutions could be related to following the modified Walden equation, but that of NaCMC solutions was not influenced by the bulk viscosity. Instead an ion "diffusion viscosity" was defined and calculated from the conductivity. It was found to correspond to the likely viscosity of the solution at a molecular scale.
\end{abstract}

\section{Keywords}

milk; whey protein; sucrose; glucose; carboxymethyl cellulose; ion mobility

\section{Introduction}

Foods such as honey, milk, and yogurt are solutions and colloidal dispersions containing carbohydrates, fat, sugars, proteins, minerals, and other minor components in water. Knowledge of physical properties is required for accurate design and simulation of processes and in this study electrical conductivity is the main property of interest, especially in the way it is affected by viscosity.

Sharifi \& Young (2012, 2013) measured the conductivity of milk solutions with up to 47\% solids and used the results for electrical tomography. They used multiple linear regression to relate the solids content and temperature to electrical conductivity, but they did not consider the effect of viscosity directly. St-Gelais et al. (1995) measured conductivity and viscosity of milk solutions as the $\mathrm{pH}$ was reduced in an attempt to monitor gelation. While their data show that both conductivity and viscosity changed, the variables appeared to be independent of each other. When the $\mathrm{pH}$ changed rapidly from 5.6 to 5.0, the viscosity increased about 100 times, while the conductivity only doubled. Henningsson et al. (2005) stated that proteins and lactose affected electrical conductivity of milk via viscosity, and that the charged proteins contributed to only $0.5 \%$ of conductivity by carrying charge. They suggested that the effect of proteins on viscosity and hence on conductivity was important.

In liquid foods, with the exception of a few such as soy sauce and fish sauce, the concentration of electrolytes is relatively low. For example concentrated skim milk with 50\% solids content will contain only about $5 \%$ salts and organic acids only some of which are present as dissociated ions (Walstra, Wouter \& Geurts, 2006), and honey has at most 2\% ash and free 
acids (White et al. 1962). Tomato ketchup which contains cellulose and starches has a maximum of about $5 \% \mathrm{NaCl}, \mathrm{KCl}$ and acids (Sharoba et al., 2005).

The starting point for most analyses is Walden's (1906) rule that proposes that electrical conductivity of a solution is inversely proportional to its viscosity. A number of researchers have studied saccharide + electrolyte systems and have proposed modifications to Walden's rule. Stokes (1959) confirmed that for $\mathrm{KCl}+$ sucrose solutions conductivity was inversely proportional to viscosity raised to the power of 0.7. Miller et al. (2000) reported powers of 0.64 for $\mathrm{NaCl}$ in trehalose and 0.78 for glucose. Longinotti and Corti (2002) gave similar results for $\mathrm{MgCl}_{2}, \mathrm{CaCl}_{2}, \mathrm{NaCl}$ and $\mathrm{KCl}$ in trehalose and sucrose, which were obtained when studying the cryoprotection properties of these solutions. They suggested changes in conductivity with concentration could be related to "preferential solvation, which in turns developed structural inhomogeneities". More data is given for various chlorides in sucrose by Mitra et al. (2010).

Acquarone et al. (2007) used electrical conductivity with $\mathrm{pH}$ at different dilutions of honey to discriminate the geographical origin of honeys. Bordi and Cametti (1986) showed that the concentration (and hence the bulk viscosity) of carboxymethyl cellulose (NaCMC) at up to 1 $\mathrm{g} / \mathrm{L}$ has little effect on electrical conductivity when the concentration of added $\mathrm{BaCl}_{2}$ was greater than about $3 \mathrm{mmol} / \mathrm{L}$. Below that concentration they used Manning ion condensation concepts to explain the effect of NaCMC concentration. The viscosities of the samples were likely to be up to 5 times that of water.

This study was conducted to establish relationship for a range of food liquids at higher concentrations and hence higher viscosities. Model solutions were prepared using sugars, whey protein, milk powder, carboxymethyl cellulose and salts, and the electrical conductivity and viscosity of these was measured.

\section{Theory}

At low concentration the conductivity, $\sigma$, of a solution can be written as a sum for different ionic species

$$
\sigma=\Sigma_{i} C_{i} \lambda_{i}
$$

where $C_{i}$ is molar concentration of ion $i$, and $\lambda_{i}$ is the ionic conductivity which in turn can be defined in terms of Faraday's number, $F$, ion charge, $z_{i}$, and ion mobility, $u_{i}$.

$$
\lambda_{i}=F z_{i} u_{i}
$$

Ion mobility can be related to diffusion and hence to viscosity, $\eta$ using the Walden equation.

$$
u_{i}=\frac{e_{0} z_{i}}{6 \pi \eta r_{i}}
$$

Here $e_{0}$ is the charge of an electron and $r_{i}$ is the Stokes' radius.

For saccharide + electrolyte systems it has been found that as the viscosity increases $\lambda \propto 1 / \eta^{\alpha}$ where $\alpha$ is a constant that has been determined to be in the range 0.64 to 0.78 for various sugars. Hence the modified Walden equation is written.

$$
u_{i}=\frac{e_{0} z_{i}}{6 \pi \eta^{\alpha} r_{i}}
$$


83 But to be dimensionally correct, the viscosity term should be written as $\eta_{0}\left(\eta / \eta_{0}\right)^{\alpha}$ where $\eta_{0}$

84 is a reference viscosity in consistent units.

85

86

87

88

89

90

91

92

94

95

96

97

98

99

100

101

102

103

For binary aqueous electrolyte solutions, as the salt concentration, $C$, increases the calculated conductivity decreases and is often described by Kohlrausch's Law

$$
\sigma=C \Lambda_{m}=\mathrm{C}\left(\Lambda_{m}^{0}-K C^{0.5}\right)
$$

where $\Lambda_{m}$ and $\Lambda_{m}^{0}$ are the molar conductivity of the solute at concentration $C$ and at infinite dilution respectively, and $K$ is a constant. Using this as a basis, and with data from Chambers, Stokes and Stokes (1956) at $25^{\circ} \mathrm{C}$ for concentrations up to $5.4 \mathrm{~mol} \mathrm{~L}^{-1}(26 \%)$, the molar conductivity of $\mathrm{NaCl}$ is given at $20^{\circ} \mathrm{C}$ by

$$
\Lambda_{M, N a C l}=\left(114.6-28.6 C_{\mathrm{NaCl}}^{0.5}\right) \frac{\eta_{w}(25)}{\eta_{w}(T)}
$$

Here molar conductivity is in units of mS.L. $\mathrm{cm}^{-1} \mathrm{~mol}^{-1}$ and concentration is in $\mathrm{mol} \mathrm{L}^{-1}$. For $\mathrm{KCl}$ up to $4 \mathrm{~mol} \mathrm{~L}^{-1}$ the equation is:

$$
\Lambda_{M, K C l}=\left(144.3-32.6 C_{K C l}^{0.3}\right) \frac{\eta_{w}(25)}{\eta_{w}(T)}
$$

These equations $(5-7)$ do not separate the effect of viscosity changes as concentration increases. Analysis of the data of Chambers et al. (1956) shows that about $90 \%$ of the drop in molar conductivity is due to the increase in viscosity over the concentration range.

van Rysselberghe and Nutting (1934) showed that adding $\mathrm{NaCl}$ and $\mathrm{KCl}$ conductivities on a molar basis was satisfactory if the component (binary) conductivities, $\Lambda_{m, i}$, are calculated at the total salt concentration, $\Sigma C_{i}$.

$$
\sigma_{m i x}=\Lambda_{m, m i x} \sum C_{i}=\sum\left(C_{i} \cdot \Lambda_{m, i}\right)
$$

Combining various equations in a more general form for a vector of concentrations in a mixed solution, $\mathbf{C}$, we can explicitly separate the effect of viscosity from the effect of increased ion concentration using the form:

$$
\sigma=\frac{f(\mathbf{C})}{\eta}
$$

104 It is proposed to use data from dilute binary solutions with similar ion concentrations as in the 105 liquid foods, but with low, known viscosities to obtain estimates of $f(C)$ for each of the 106 components in a solution and combine these using an equation of the form of (8). In food 107 systems the exact composition is often unknown so it is intended obtain an effective concentration of all salts lumped together as single equivalent salt such as $\mathrm{NaCl}$.

110 It is known that the inverse relationship with viscosity in Equation (3) will fail when solutions are no longer dilute, but rather than use the modified Walden equation (4), it is proposed that an effective viscosity be determined. This will be the viscosity experienced by conducting ions as they diffuse under the influence of the electric field. In this work it is termed the "diffusion 114 viscosity", $\eta_{\text {diff }}$, which can be estimated from the known ion concentrations and the measured 115 conductivity.

$$
\eta_{\text {diff }}=\frac{f(\mathbf{C})}{\sigma}
$$


117 The bulk viscosities, $\eta$, of binary solutions of glucose, fructose and sucrose were required to 118 calculate conductivities using the modified Walden equation (4). Data were obtained from 119 Weast (1977) and equations were fitted in the form of Equation (11) recommended by Morison 120 and Hartel (2007) to obtain constants.

$$
\eta(T)=\eta_{w}(T) e^{\sum_{i} a_{i} \frac{w_{i}}{w_{w}}+b_{i}\left(\frac{w_{i}}{w_{w}}\right)^{2}}
$$

121

122

123

124

125

126

127

128

129

130

131

132

133

134

135

136

137

138

139

140

141

142

143

144

145

146

Here $T$ is the temperature, $w$ is mass fraction, $a$ and $b$ are temperature dependent constants for a binary solution, subscript $i$ refers to a single component $i$ and subscript $w$ refers to water. Table 1 gives a set of coefficients for this equation.

Table 1. Temperature $\left({ }^{\circ} \mathrm{C}\right)$ dependent coefficients for viscosity using Equation (11)

\begin{tabular}{lcc}
\hline Component & $\boldsymbol{a}$ & $\boldsymbol{b}$ \\
\hline Sucrose & $3.165-0.01956 T+6.9 \times 10^{-5} T^{2}$ & $0.0063-0.0037 \mathrm{~T}+2.2 \times 10^{-5} T^{2}$ \\
Glucose & $2.829-0.0126 T-1.2 \times 10^{-4} T^{2}$ & $0.0097-0.0045 T$ \\
Fructose & $2.764-0.0177 T+9.4 \times 10^{-5} T^{2}$ & $0.082-0.0064 T$ \\
\hline
\end{tabular}

\section{Materials and Methods}

Skimmed milk solids purchased from a local supermarket (Alpine brand, 55\% lactose, 33\% protein, $1 \%$ fat, $7 \%$ minerals, $4 \%$ water) was used to prepare milk solutions. A stick homogenizer was used to disperse the milk solids in Milli-Q water in the preparation of milk solutions. The solutions were held at $45^{\circ} \mathrm{C}$ for 40 minutes to enable hydration. Binary solutions of glucose, fructose and sucrose were prepared from food grade sugars. Sodium carboxymethyl cellulose (NaCMC) (Walocel C, DOW) solutions were prepared at concentrations up to $1 \%$ by mass of NaCMC powder (wet basis). The powder was dried at $105{ }^{\circ} \mathrm{C}$ and found to contain $8.5 \%$ water. In addition various concentrations of $\mathrm{KCl}, \mathrm{NaCl}$ and sucrose were added. Solutions of whey protein isolate (WPI) (Balance Sports Nutrition, New Zealand), with and without lactose and $\mathrm{NaCl}$ were also prepared. Weighing was achieved with an accuracy better than 1 $\mathrm{mg}$ in $100 \mathrm{~g}$ of solution. The set of solutions tested is given in Table 2. The preparation and conductivity of NaCMC solutions was repeated two months after the first set.

Table 2. Composition of solutions

\begin{tabular}{llll}
\hline Main component & Mass fraction & Added component & Mass fraction \\
\hline NaCMC & $0-1 \%$ & & \\
\hline NaCMC & $0-1 \%$ & $\mathrm{NaCl}$ & $0.01 \%$ \\
\hline NaCMC & $0-1 \%$ & $\mathrm{KCl}$ & $0.01 \%$ \\
\hline WPI & $0-1 \%$ & $\begin{array}{l}\text { Sucrose } \\
\text { KCl }\end{array}$ & $\begin{array}{l}30 \% \\
0.013 \%\end{array}$ \\
\hline WPI & $1,10,20,30 \%$ & & $0.01 \%, 0.1 \%, 1 \%$ \\
\hline WPI & $20 \%$ & $\mathrm{NaCl}$ & $20 \%$ \\
& $20 \%$ & Lactose & $0.01 \%, 1 \%$ \\
\hline Fructose & & $\mathrm{NaCl}$ & \\
\hline Glucose & $0.01 \%-55 \%$ & & \\
\hline Sucrose & $0.01 \%-60 \%$ & & \\
\hline Skim milk reconstituted & $0.1 \%-70 \%$ & & \\
\hline
\end{tabular}

The electrical conductivity of the solutions were measured using a Schott conductivity probe LF413T, with an electrode gap of about $1 \mathrm{~cm}$, connected to a Schott Lab 960 benchtop meter, the same probe was connected to a FLUKE PM6306 programmable RCL meter operating at 
$0.1 \mathrm{~V}$ a.c.. The RCL meter was used because the benchtop meter had a minimum resolution of

$148 \quad 0.1 \mu \mathrm{S} / \mathrm{cm}$ which was insufficient at the low conductivities measured in concentrated sugar solutions. A custom-made sample holder was used to seal the conductivity probe in place and to provide consistent experimental conditions. The container was immersed in a water bath at $20.0^{\circ} \mathrm{C}$ with control of $\pm 0.02{ }^{\circ} \mathrm{C}$. The temperature was measured with a precision platinum thermometers with an accuracy of $\pm 0.01^{\circ} \mathrm{C}$. The resistance at a frequency with zero or near zero phase angle was selected for the conductivity calculations. This reduced the influence of any capacitance or inductance in the measurement system. For low conductivity solutions the best frequency was about $100 \mathrm{~Hz}$, but it was as high as $70 \mathrm{kHz}$ for highly conductive solutions. The error introduced by doubling or halving the frequency was less than $2 \%$ is all cases, indicating low sensitivity to frequency. The cell was calibrated using standard $\mathrm{KCl}$ solutions with different mass fractions (Shreiner and Pratt, 2004) with a maximum deviation of $1 \%$. Repeated measurements were found to be always consistent to better than the larger of $0.5 \%$ or $1.5 \mu \mathrm{S} / \mathrm{cm}$. When the temperature deviated from $20.0^{\circ} \mathrm{C}$, by at most $0.02{ }^{\circ} \mathrm{C}$, the measured resistance were corrected to $20.0{ }^{\circ} \mathrm{C}$ using a measured temperature coefficient of $2 \%{ }^{\circ} \mathrm{C}^{-1}$. Repeated calibration of the conductivity and viscosity measurements showed no measureable drift.

For all solutions, a Haake Rotovisco RV20 with an NV cylinder set was used to measure the viscosities at $20.0^{\circ} \mathrm{C}$. The Haake viscometer was calibrated using a Cannon N1000 calibration oil to within $\pm 1 \%$ over the rotational speed range of the viscometer. At absolute uncertainty was estimated from water to be better than $0.3 \mathrm{mPa}$.s. Repeated measurements were always less than $2 \%$ different. The apparent viscosities are given at a shear rate of $2700 \mathrm{~s}^{-1}$.

\section{Results and Discussion}

The relationship between conductivity and viscosity with concentration for four different conductivity is variable. The conductivity of the NaCMC solution (Figure 1a) is linearly related to concentration and is only slightly affected by the increasing viscosity. The conductivity increases due to the sodium counter-ions and also the residual $\mathrm{NaCl}$ normally present in NaCMC. The long NaCMC molecules form a tangled network which increases the bulk viscosity, but within which ions can freely move. The molecular mass of the NaCMC was unknown but it typically about $10^{5} \mathrm{~g} / \mathrm{mol}$.

At the other extreme is sucrose (Figure 1d) (and other sugars) which show a marked decline in conductivity as the viscosity rises. This has been well studied as stated in the Introduction. The modified Walden equation was fitted giving a value of $\alpha=0.645$ and a dry basis salt content in sucrose of $39 \mathrm{ppm}$ as $\mathrm{NaCl}$ equivalent. The value of $\alpha$ fits well within the range $0.64 \pm 0.02$ given Miller et al. (2000) for disaccharides. The error bars in Figure 1d were determined from the largest uncertainties found when repeating conductivity measurements of NaCMC, but the deviation from a smoothed curve indicates much lower uncertainties. The uncertainties in other graphs are mostly smaller than the symbols and are shown only in Figure 1a.

The WPI solution (Figure 1b) is similar to NaCMC but there is a reduction in the slope of the conductivity curve. The whey proteins in WPI are $\beta$-lactoglobulin and $\alpha$-lactalbumin, with molecular masses of 18400 and $14200 \mathrm{~g} / \mathrm{mol}$ respectively, and are small enough to interact more with ions thus reducing their mobility. Skim milk concentrate (Figure 1c) contains about $50 \%$ lactose and $40 \%$ protein on a dry basis so the resulting conductivity shows behaviour intermediate between the WPI solution and sucrose solution. The near-constant conductivity above $25 \%$ solids is most likely caused by the coincidental opposite effects of increased ion 
197 concentration and increased viscosity. The conductivities of skim milk solutions were very 198 similar to those obtained by Sharifi and Young (2012).

199

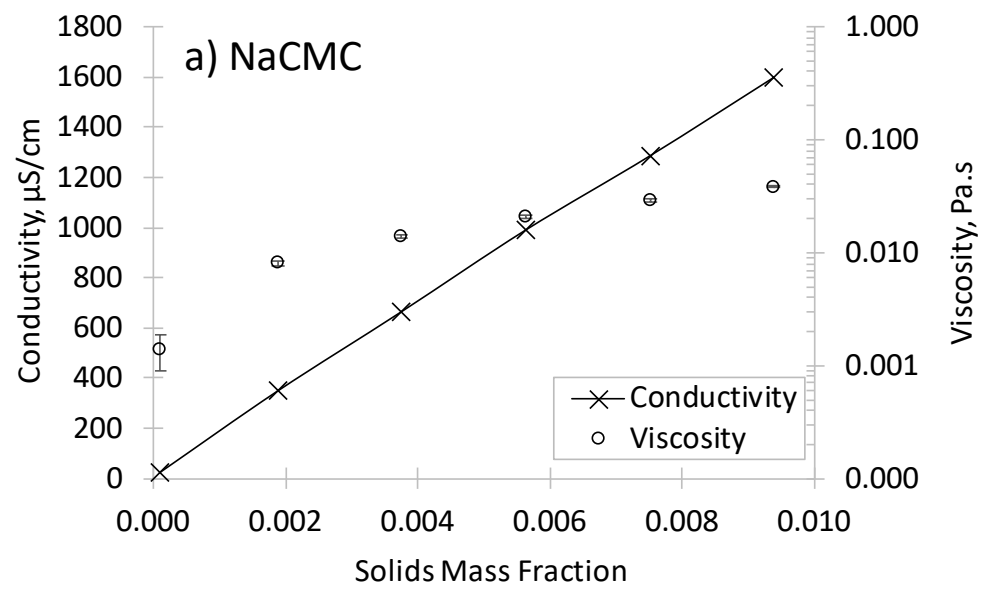

200

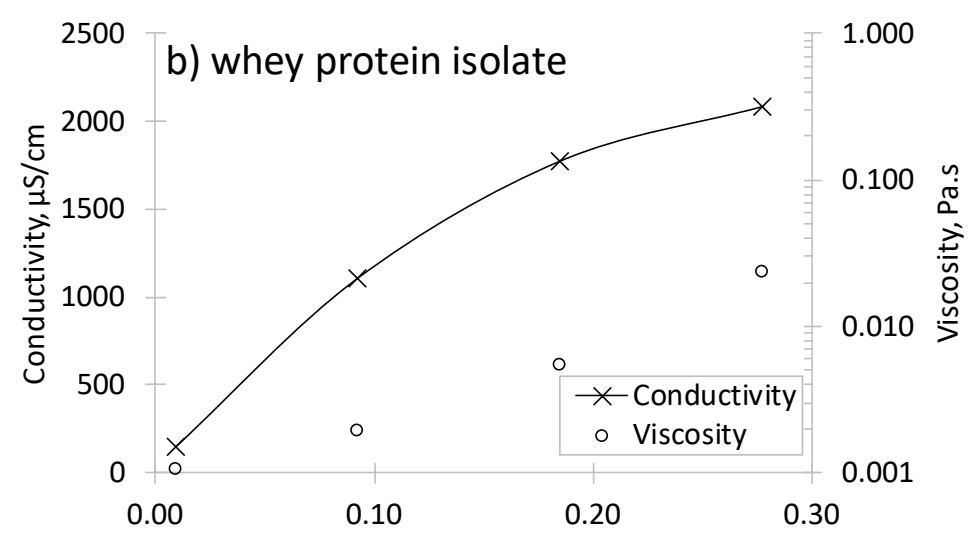

201

Solids Mass Fraction

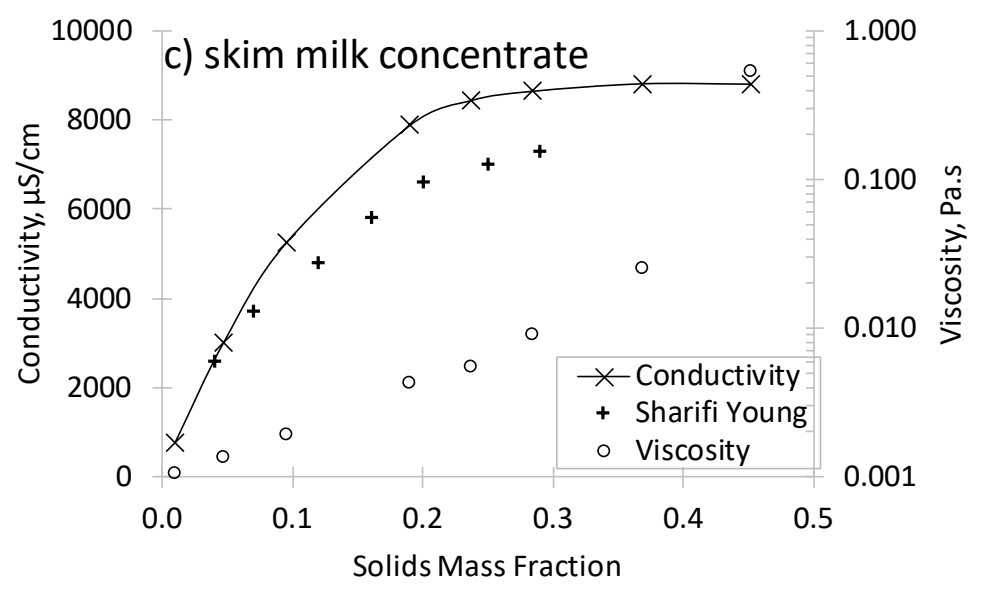




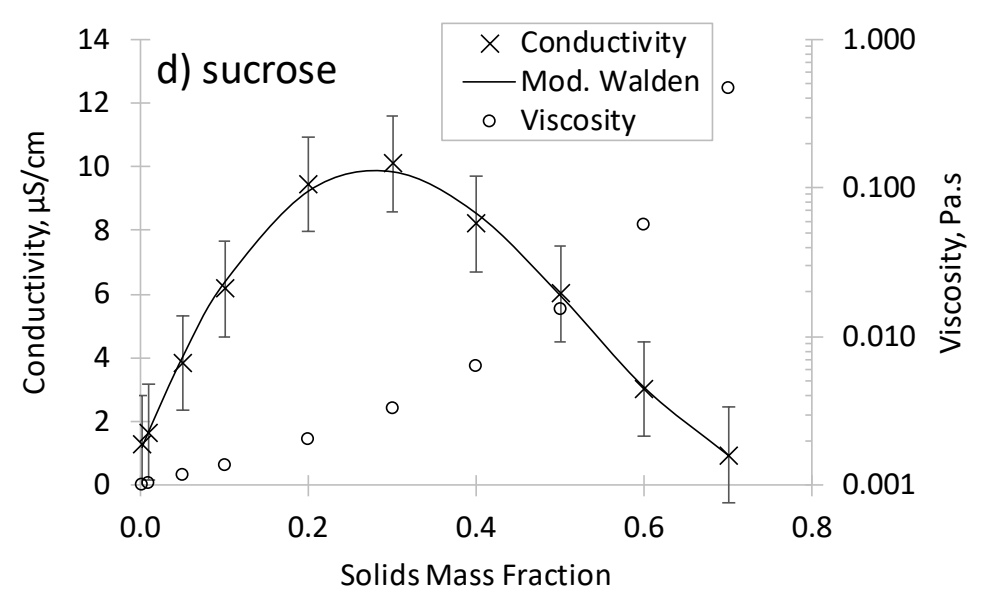

Figure 1. Electrical conductivity and viscosity of four different solutions: NaCMC, whey protein isolate, skim milk powder and sucrose. Conductivities for skim milk solutions from Sharifi and Young (2012) are included in c). The best fit using the modified Walden equation is shown for sucrose in d). Note the logarithmic scale for viscosity.

If the modified Walden Equation (4) is applied to NaCMC, a best fit is obtained using $\alpha=$ 0.03 , which effectively shows the insignificant contribution of bulk viscosity. It was concluded that Equation (4) is not suitable for NaCMC. Likewise it did not work for WPI and milk solutions.

As an alternative approach the "diffusion viscosity" was calculated using Equation (10). First, using $\mathrm{NaCMC}+\mathrm{KCl}$ as an example, the equivalent concentration of $\mathrm{NaCl}$ in $\mathrm{NaCMC}$ was fitted. The conductivity of the equivalent solution of $\mathrm{NaCl}$, as plotted in Figure 2, was fitted using quadratic equations to have the same slope of conductivity as the NaCMC at zero concentrations (where there was negligible viscosity effect). This yielded an equivalent concentration of $\mathrm{NaCl}$ of $10.9 \%$ dry basis in NaCMC powder. The solution of $0.6 \% \mathrm{NaCMC}$ $+0.01 \% \mathrm{KCl}$ was then considered to be equivalent to a solution of $0.006 \times 0.109=0.000672$ mass fraction of $\mathrm{NaCl}+0.0001$ mass fraction of $\mathrm{KCl}$. The conductivity of a mixture of $\mathrm{NaCl}$ and $\mathrm{KCl}$ at these concentrations was calculated from Equations (6) to (8) to be $1189 \mu \mathrm{S} \mathrm{m}^{-1}$ and the product of the conductivity and viscosity, $f(C)$, of a solution of $\mathrm{NaCl}+\mathrm{KCl}$ only was $1.192 \mu \mathrm{S} \mathrm{Pa} \mathrm{s} \mathrm{cm}{ }^{-1}$. The measured conductivity was $1151 \mu \mathrm{S} \mathrm{cm} \mathrm{cm}^{-1}$ at $20{ }^{\circ} \mathrm{C}$. Hence using Equation (10) the diffusion viscosity was calculated to be $1.192 / 1151=0.00104$ Pa.s (only slightly higher than water).

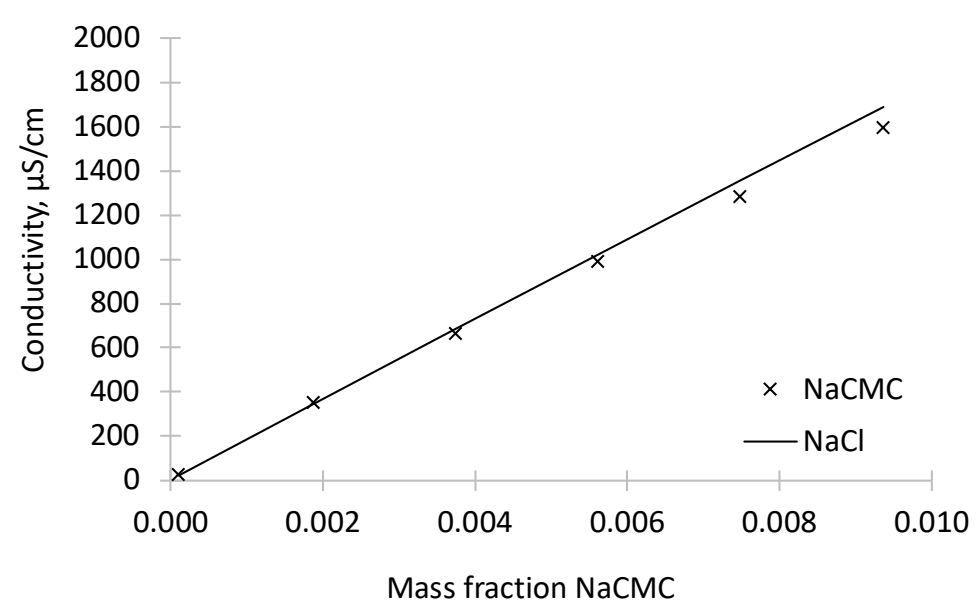

Figure 2. Conductivity of NaCMC solution and of an equivalent solution of $\mathrm{NaCl}$ fitted to have the same slope at zero concentration. 
233 The diffusion viscosity was calculated and shown in Figure 3. The first points corresponded to a low conductivity with corresponding large experimental error. The viscosity "experienced" by the conducting ions is shown to be only slightly greater than that of water. Figure 3 shows two different sets of results obtained two months apart, indicating low experimental uncertainty, except at very low concentration where the solution conductivity had greater measurement error.

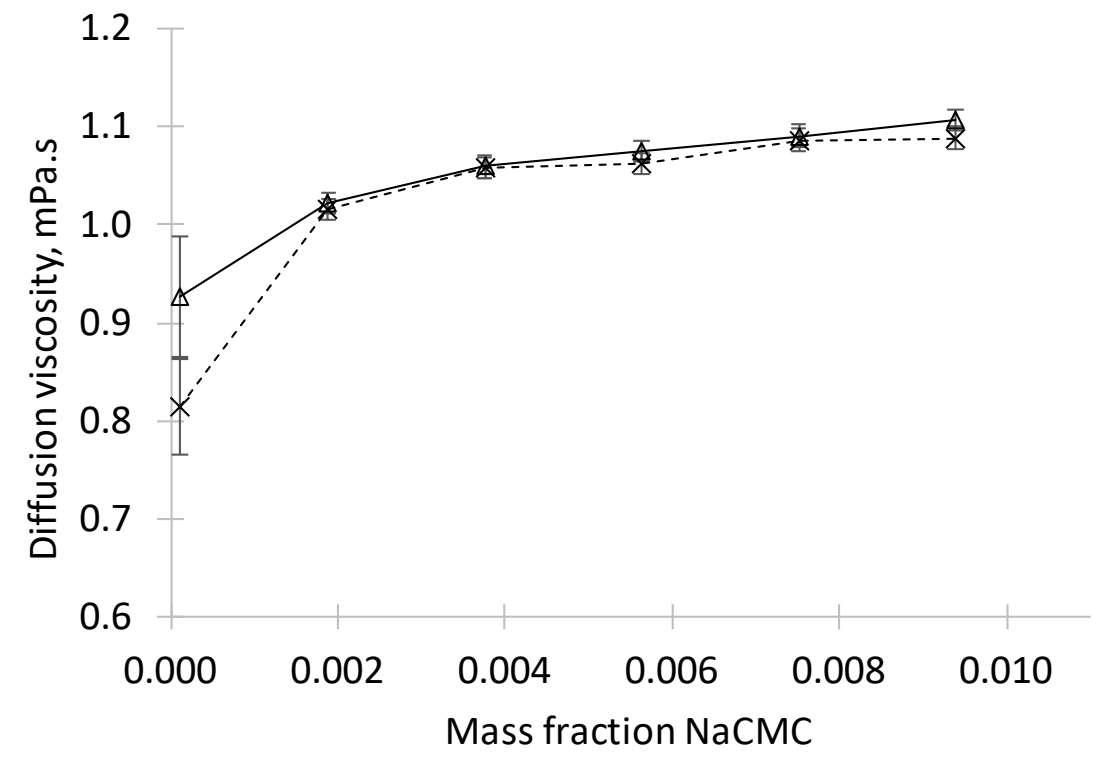

Figure 4 shows the result of similar calculations for other mixtures. For comparison, the results for sucrose are included in each graph. The graphs show good consistency between similar solutions, but quite different results for different solutions. The diffusion of ions through NaCMC solutions, with and without salts, seems only slightly affected by mass fraction even though, as seen in Figure 1a, the bulk viscosity increases significantly. Here the mass fractions are very low because higher concentrations are difficult to achieve. The diffusion viscosities for WPI and skim milk are higher than for sucrose at similar concentrations, showing greater resistance to ion flow. This is contrary to expectations as WPI and milk solutions have a lower bulk viscosity than sucrose at the same concentration. It is likely that there is significant interaction between ions and the protein which will have a negative charge in water. The different sugars show similar behaviour to each other. 

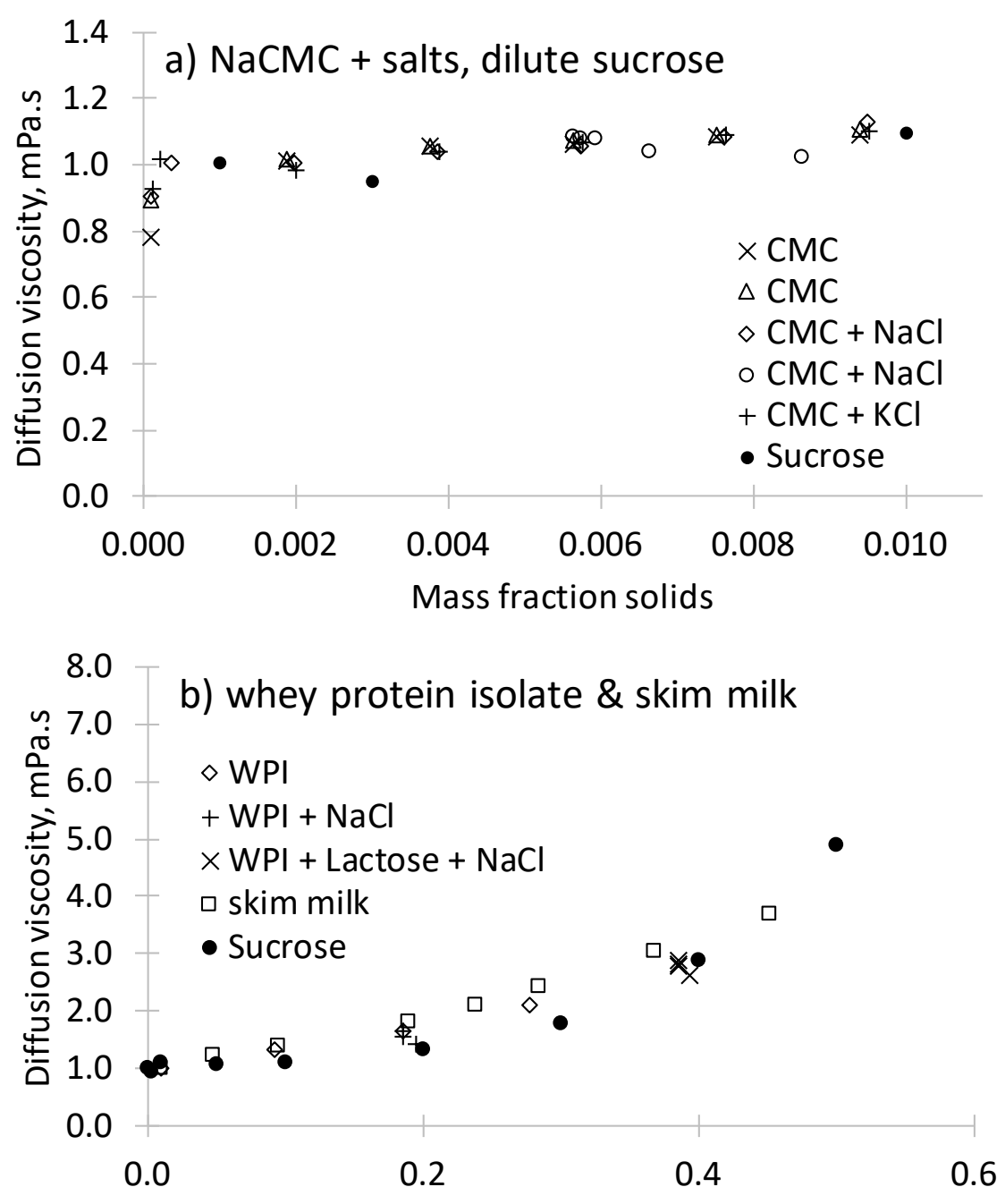

Mass fraction solids

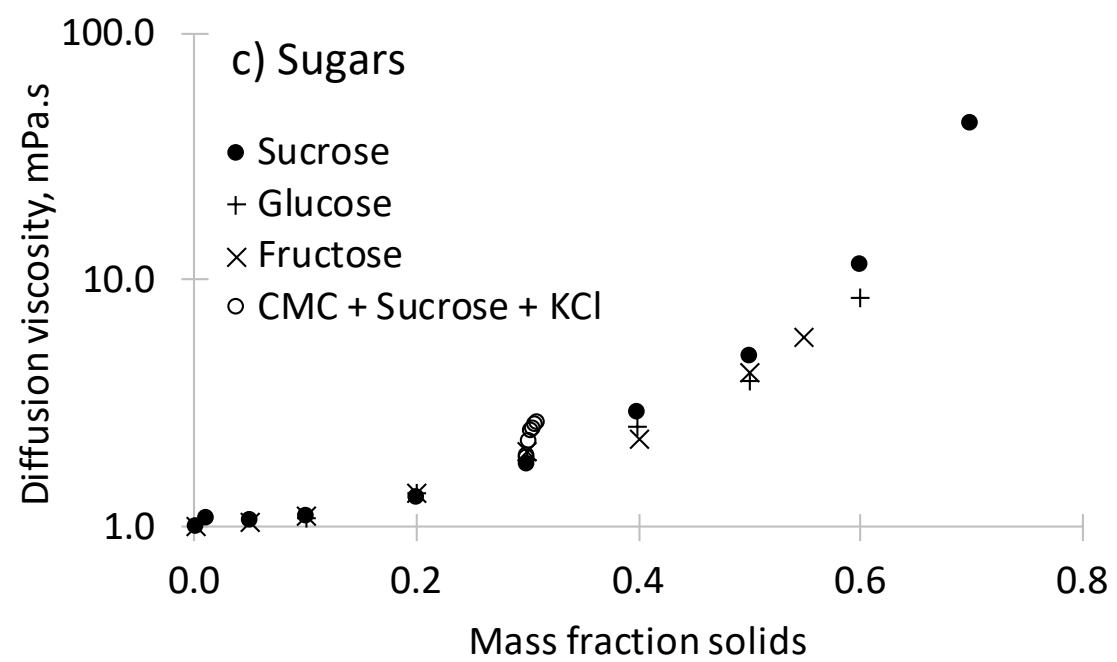

The ratio of the diffusion viscosity to the bulk viscosity was calculated and is shown in Figure 5. These graphs clearly show that the bulk viscosity is not a good measure of the viscosity experienced by conducting ions as the ratio is significantly less than 1.0. The indicative error bars of two series show the influence of poor accuracy at low bulk viscosities because of the 
accuracy limits of the Haake viscometer at these low viscosities. The relationships for sugars, WPI and skim milk between the ratio and solids content are surprisingly linear with concentration, but given that the relationship involves a number of non-linear equations, this is more likely to be coincidental than fundamental.

269
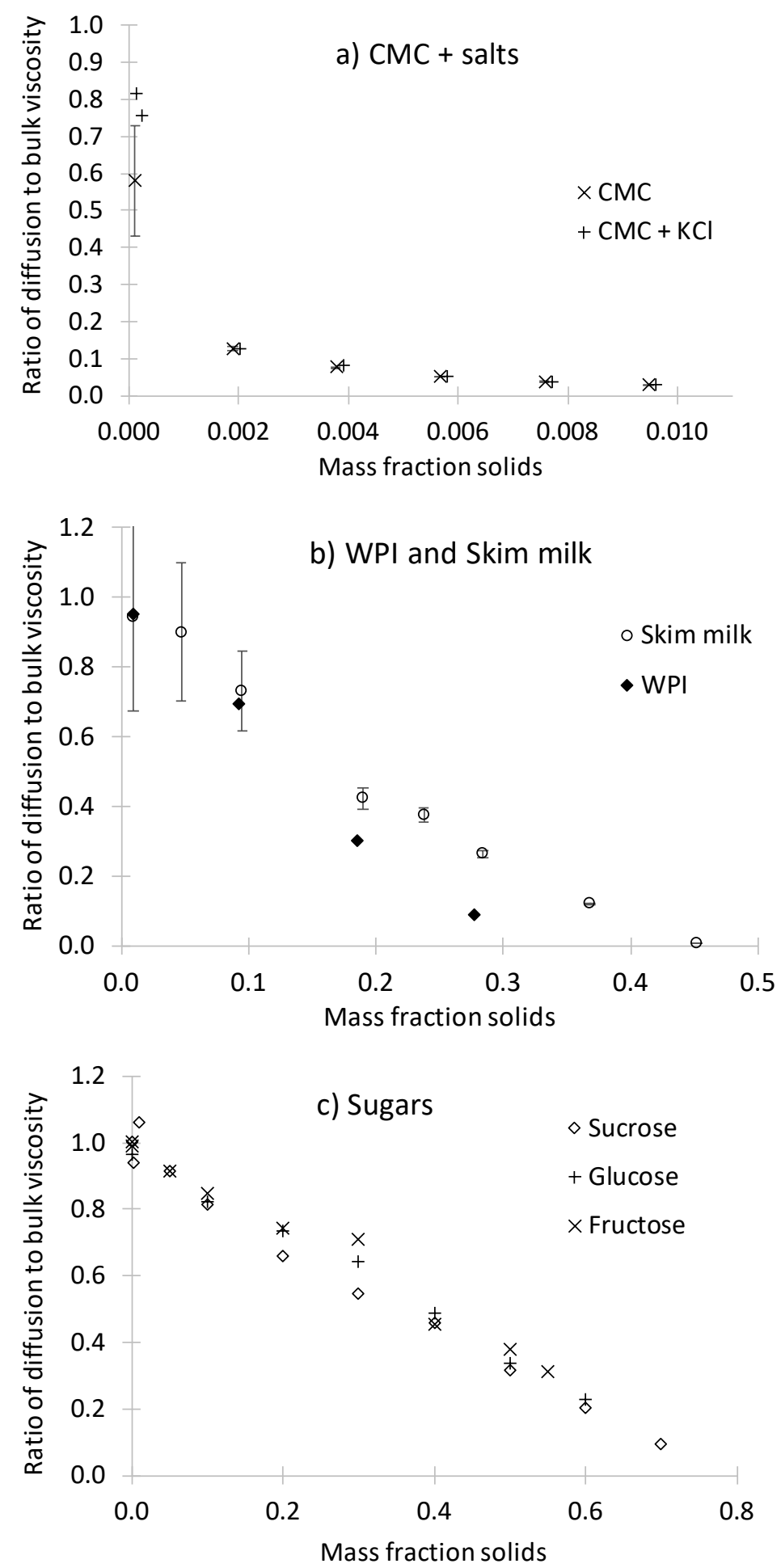

Figure 5. The ratio of calculated diffusion viscosity to bulk viscosity for various solutions. Error bars on two of the series indicate calculated uncertainties. 
The results presented so far do not lead to a general prediction equation for electrical conductivity. The modified Walden's rule can be applied to sugar solutions, but the effect of bulk viscosity can probably be ignored in solutions of long chain polymers such as NaCMC. The conductivities of other solutions containing large molecules could not be predicted $a$ priori.

For a specific type of liquid it is recommended that the conductivity be related to the diffusion viscosity so that empirical relationships for prediction of conductivity can be determined. However, for a better understanding of ion transport, interactions at a molecular scale could be investigated. For charged molecules like NaCMC and other polyelectrolytes, better predictions for a specific liquid could be obtained by considering that state of the charge and counter-ions, perhaps over a range of $\mathrm{pH}$ values. In contrast sugars have minimal interactions with ions and hence a model based on friction between species and tortuous ion diffusion (Krishna and Wesselingh, 1997) might be effective at high concentrations.

When the solids content was low $(<10 \%)$, the diffusion viscosity was not much greater than that of water, even though the bulk viscosity could be high. Hence the conductivity was mostly dependent on the ion concentration in solution.

\section{Conclusions}

The application of the modified Walden equation to liquid foods other than sugars was unsuccessful. Experimental results showed that, in general, electrical conductivity was not well correlated with bulk viscosity. Conducting ions were able to pass through viscous NaCMC solutions with little extra resistance. It seems likely that electrical conductivities will be unaffected by the bulk viscosity when the viscosity is high because of entanglement of long polymer chains.

302

303

In liquids such as concentrated sugar solutions and honey, in which the viscosity is dominated by small molecules, the electrical conductivity is strongly affected by viscosity. However as already found by many researchers, conductivity of many viscous solutions is not directly related to the inverse of the bulk viscosity.

Instead it was found that a diffusion viscosity could be determined from conductivity data. This showed the resistance to ion movement and gave greater insight into the effects of composition on conductivity.

In milk and whey protein solutions there is some evidence of interaction between ions and the charged proteins which slows the movement of ions and this results in a higher than expected diffusion viscosity. A more complete model including the ion/protein interaction could account for the observed change. Experiments in which protein charge is changed by altering the $\mathrm{pH}$ should allow this effect to be quantified.

\section{Acknowledgement}

Author Subbiah was supported by a University of Canterbury Doctoral Scholarship.

\section{References}

322 Acquarone, C., Buera, P., Elizalde, B., 2007, Pattern of pH and electrical conductivity upon

323 honey dilution as a complementary tool for discriminating geographical origin of honeys,

324 Food Chemistry 101, 695-703. 

solutions with divalent counterions, Journal of Physical Chemistry 90, 3034-3038.

Chambers, J.F., Stokes, J.M., Stokes, R.H., 1956, Conductances of concentrated aqueous sodium and potassium chloride solutions at $25^{\circ}$. Journal of Physical Chemistry 60, 985-986.

Henningsson, M., Ostergren, K., Petr Dejmek, 2005, The electrical conductivity of milk - the effect of dilution and temperature, International Journal of Food Properties, 8, 15-22.

Krishna, R.; Wesselingh, J.A., 1997, The Maxwell-Stefan approach to mass transfer, Chemical Engineering Science, 52, 861-911.

Longinotti, M.P., Mazzobre, M.F., Buerax, M.P., Corti, H.R., 2002, Effect of salts on the properties of aqueous sugar systems in relation to biomaterial stabilization. Part 2. Sugar crystallization rate and electrical conductivity behavior, Phys. Chem. Chem. Phys., 2002, 4, 533-540.

Miller, D.P., Conrad, P.B., Fucito, S., Corti, H.R., de Pablo, J.J., 2000, Electrical conductivity 338 of supercooled aqueous mixtures of trehalose with sodium chloride, J. Phys. Chem. B, 104, 339 10419-10425.

340 Mitra, S.K., Kumar, V., Sanyal, P., 2010, Conductometric behaviour of salts in sugar solution, 341 Sugar Tech 12(2) 115-119.

Morison, K.R., Hartel, R.W., 2007, Evaporation and freeze drying in Heldman, D.R.; Lund, D.B. "Handbook of Food Engineering", 2nd ed., CRC Press: Boca Raton, FL, 495-552.

Sharoba, A.M., Senge, B. El-Mansy, H.A., Bahlol, H.E., B Blochwitz, R., 2005, Chemical, sensory and rheological properties of some commercial German and Egyptian tomato ketchups, European Food Research and Technology, 220, 142-151.

Shreiner, R.H., Pratt, K.W., 2004, Standard Reference Materials: Primary Standards and Standard Reference Materials for Electrolytic Conductivity, NIST Special Publication 260142.

Sharifi, M., Young, B., 2012, Milk total solids and fat content soft sensing via electrical resistance tomography and temperature measurement, Food and Bioproducts Processing, 90, 659-666.

353 Sharifi, M., Young, B., 2013, Towards an online milk concentration sensor using ERT:

354 Correlation of conductivity, temperature and composition, Journal of Food Engineering, 116, 355 86-96.

356 St-Gelais, D., Champagne, C. P., Erepmoc, F., Audet, P., 1995, The use of electrical 357 conductivity to follow acidification of dairy blends, International Dairy Journal, 5, 427-438.

358 Stokes, R. H., 1959, Mobilities of ions and uncharged molecules in relation to viscosity - a 359 classical viewpoint, in The Structure of Electrolytic Solutions; Hamer, W. J., Ed.; Wiley: New 360 York, Chapter 20, p 298.

361 Van Rysselberghe P, Nutting L., 1934, The conductivity of concentrated mixtures of alkali 362 chlorides, Journal of the American Chemical Society. 56: 1435-1437.

Walden, P., 1906, Über organische Lösungs-und Ionisierungsmittel. III. Teil: Innere Reibung und deren Zusammenhang mit dem Leitvermögen. Z. Phys. Chem. 55, 207-246.

Walstra, P., Wouters, J.T.M., Geurts, T.J., 2006, Dairy Science and Technology, $2^{\text {nd }}$ ed., CRC Press, Boca Raton. 
367 Weast, R.C. (ed), 1977, CRC Handbook of Chemistry and Physics, 58 ${ }^{\text {th }}$ ed. CRC Press, Florida.

368 White, J.W., Jr., Reitof, M.L., Subers, M.H. \& Kushnir, I. (1962). Composition of

369 American Honeys. Tech. Bull. U.S. Dep. Agric., No 1261, 124. 